\title{
OSCONVR: An Interactive Virtual Reality Interface to an Object-Oriented Database System for Construction Architectural Design
}

\author{
Farhi Marir, Karim Ouazzane, and Kamel Zerzour \\ Knowledge Management Group (KMG), School of Informatics and Multimedia \\ Technology, University of North London, Holloway Road, London N7 8DB, UK \\ \{f.marir, k.ouazzane, k.zerzour\}@unl.ac.uk
}

\begin{abstract}
The paper presents the OSCONVR system, which is an interactive interface to an Object database for construction architecture design. The OSCONVR system is part of the OSCON (Open Systems for Construction) funded project. The aim of the OSCONVR system is to explore the potentials of using virtual reality as an interface for an integrated project database using the World Wide Web. It describes the steps taken to link the OSCON integrated database to a VRML environment.

Virtual Reality has often been looked at as a visualisation tool. This paper puts the argument that VR should be used as the user interface. For instance, the user should interact with a 3D column in VR rather than a column in traditional database environment. This will allow the construction practitioners better access to information, which will motivate them to use integrated databases. In addition, VR should be used as a vehicle for classifying information. The user should be able to look at the design, costing, time planning, and facilities management views according to his/her requirements. This may prove helpful in projecting information from a project integrated database.
\end{abstract}

\section{Introduction}

The need for remote accessing of information within an integrated environment has become a necessity in this changing world. Information needs to be queried and interrogated in order to assist the various professions in the construction sector. For instance, site engineers should be able to access and query the remote database from their sites if they have access to a modem and the Internet. Technologies are now available which can be used to this end. The Internet and its facilities should be exploited for the benefits of better management and retrieval of construction information. The VRML (Virtual Reality Modelling Language) which is a web-based standard will be explored as a means of remotely interrogating information stored within an integrated database. The paper highlights the main findings of the OSCON integrated database project, which has managed to link the database to the VRML environment [3]. A VRML application, which reads information about design produced in AutoCAD, has been developed within the OSCON project. The VRML over the Internet will allow practitioners within the construction industry better access to the OSCON integrated database. The main benefits associated with the use of 
VRML are the integration of project databases and VRML technologies, which will ultimately result in better productivity through the effective retrieval of information.

This paper aims at demonstrating the benefits of using the Internet to access construction information. The VRML facility will be used to demonstrate how information can be remotely accessed from a database. In order to achieve this, a VRML-based prototype has been developed. Common Gateway Interface programs can then be embedded in web pages. When the page is accessed, the program is automatically executed on the server and results are sent over the net to the user. These facilities are freely available on the web. If exploited properly, the construction industry will benefit tremendously in terms of information exchanges and management. It is expected that the user will be able to query information about specifications, design information, cost estimating and time planning information. In addition, he/she will be able to access information about suppliers and materials, which are freely available on the web.

\section{Virtual Reality and Architecture Design}

One of the major problems associated with construction project integrated databases is the shear information involved and the complexity of storing such information. Traditionally, the user interface provided within databases is used to query information stored in these databases. This may prove difficult in browsing through the many records of instances of entities developed within the scope of a certain construction application. One way of browsing and querying is through the use of VR interfaces. It is a more natural way of interfacing with information as the user can visually identify the objects of interests and retrieve information about them using the VR interface. This approach was adopted by OSCON in order to respond to the user needs for a more user friendly environment. Within OSCON, the user can navigate through the VR model and identify elements by clicking on them. Information is then obtained about the specific objects depending on the view or requirements of a particular participant of the construction process. For instance, the designer can retrieve information about the specifications of a cavity wall, the QS can obtain cost information about the cavity wall, and the time planner queries the model about duration of building the cavity wall. This process will be described in detail in one of the forthcoming sections. To conclude, this paper suggests that VR technologies should be used as means of providing better information interface, in addition to their visualisation capabilities.

An awareness workshop on VR and rapid prototyping for engineering was organised in the UK by the EPSRC (Engineering and Physical Sciences Research Council) has highlighted the benefits of using VR as the technology for visualisation and interactions with $3 \mathrm{D}$ models $[2,10,13]$. The proceedings of this workshop include some of the most up to date literature within VR in the UK. Most of the papers discuss how VR can be used as design tools [4]. Animation and simulation was another theme $[14,12]$. Training was also mentioned where VR can play a major role. Alshawi \& Faraj [1] was amongst the few researchers who suggested that VR should be used as the interface for $3 \mathrm{D}$ models and databases. This paper builds on the work 
done by the previous researchers in order to develop a VR tool, which can be used as a combination of visualisation, animation and simulation and as an interface for an object oriented integrated database. The user should be able to interact with objects rather than using DXF files as suggested by Alshawi and Faraj [1] and Griffin [4]. Some useful web sites are included in the references $[5,6,7,8,9]$.

\section{The OSCONVR System}

The OSCONVR work is part of the OSCON funded project, which aims at the development of a framework for integration of construction information. The integrated database within OSCON supports the functions of design, estimating and planning by allowing these phases to effectively share information dynamically and intelligently. The system revolves around a central object-oriented information model. This model consists of domain models, which support integration of information within a specific domain, e.g. estimating. The information model also contains a core model, which captures knowledge about the means of transferring information across domains. All the models in the system are fully independent of specific applications, and each domain model provides support for general classes of a given application. In order to demonstrate how an integrated approach can benefit construction projects, the OSCON team has developed a suite of software applications, e.g. CAD application, cost estimating and planning, wrapper software for CA-Superproject ${ }^{\circledR}$, a VRML interface which will actively share construction information via a central object-oriented project database as shown in Figure 1. The CAD application allows a user to create and manipulate architectural components of a building. The components are stored as instances of classes in the object oriented database. These instances are read by the VRML interface in order to create a 3D view of the building which gives the user a better environment for navigation and walkthrough. Time and cost estimates are also generated automatically based on the design information stored in the database. The applications are being developed on PCs running under Microsoft Windows NT and are implemented in Microsoft Visual C++. The database is implemented using ObjectStore ${ }^{\circledR}$ OODBMS in conjunction with Object Engineering Workbench (OEW®) modelling software. OEW is useful for generating OSCON models and the associated code in $\mathrm{C}++$.

The integration approach developed by OSCON is generic and thus adaptable to any specific requirements of the industry. The system can, for example, be easily tailored to solving problems within the civil engineering or any other industry. This system can enhance the efficiency of the industry as a whole, improve productivity and consequently speed up the process of design and construction through the rapid prototyping facilities provided by the OSCON database.

The overall architecture of the OSCON system is shown below. 


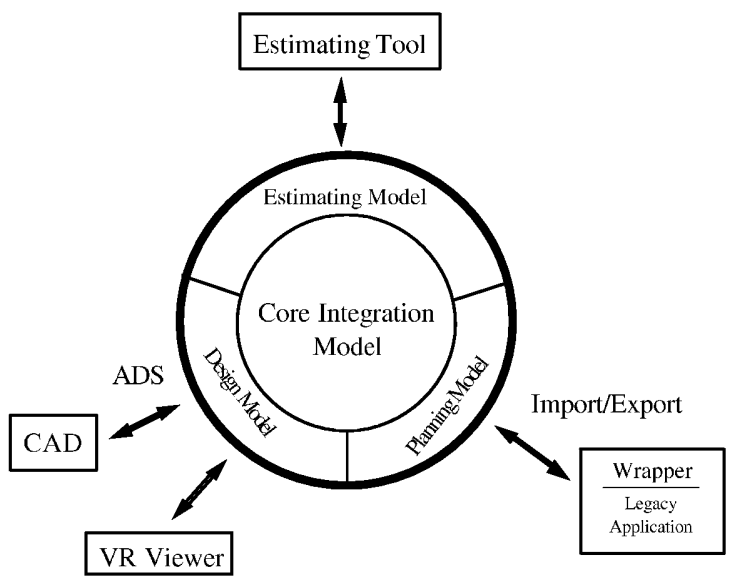

Fig. 1. The OSCON Overall architecture

\subsection{OSCONVR Architectural Design Model}

The root cause of the different problems in construction projects is the information or data upon which all participants depend. Because of fragmentation within the industry, there are many different interpretations of the semantics of the data in use. The formal ways in which to describe information semantics are referred to as models, which represent a formal description of a view of a domain of interest. Several researchers have suggested models for space and space enclosure and have also developed object-oriented product models that could be used to store richer kinds of data and knowledge about a product, including knowledge about its design, manufacturing and operational parameters [15]. None of these models has a universal acceptance. This is due to the fact that a data model must be readily accessible to all construction-related applications and users. It must also be compatible with systems across AEC and other industry [16]. Recently a group of software companies and users have come together to form the Industry Alliance for Interoperability and define specifications for a set of Industry Foundation Classes [17]. The aim of the OSCONVR system is not to recommend another design model but to derive classes from the IFC Generic classes and enhance them with knowledge about the detailed design stage that can be shared with other applications, such as cost and time planning. The detailed design model is a description of the design components and their specifications with very limited topological properties incorporated as shown in Figure 2. The model offers the representation of several structural members:

- Foundation: Raft, pad and strip.

- Slab: Ground slab, floor slab

- Columns,

- Beams, 
- Walls: solid and cavity walls,

- Roof, and

- $\quad$ Non-structural members, such as Windows and Doors.

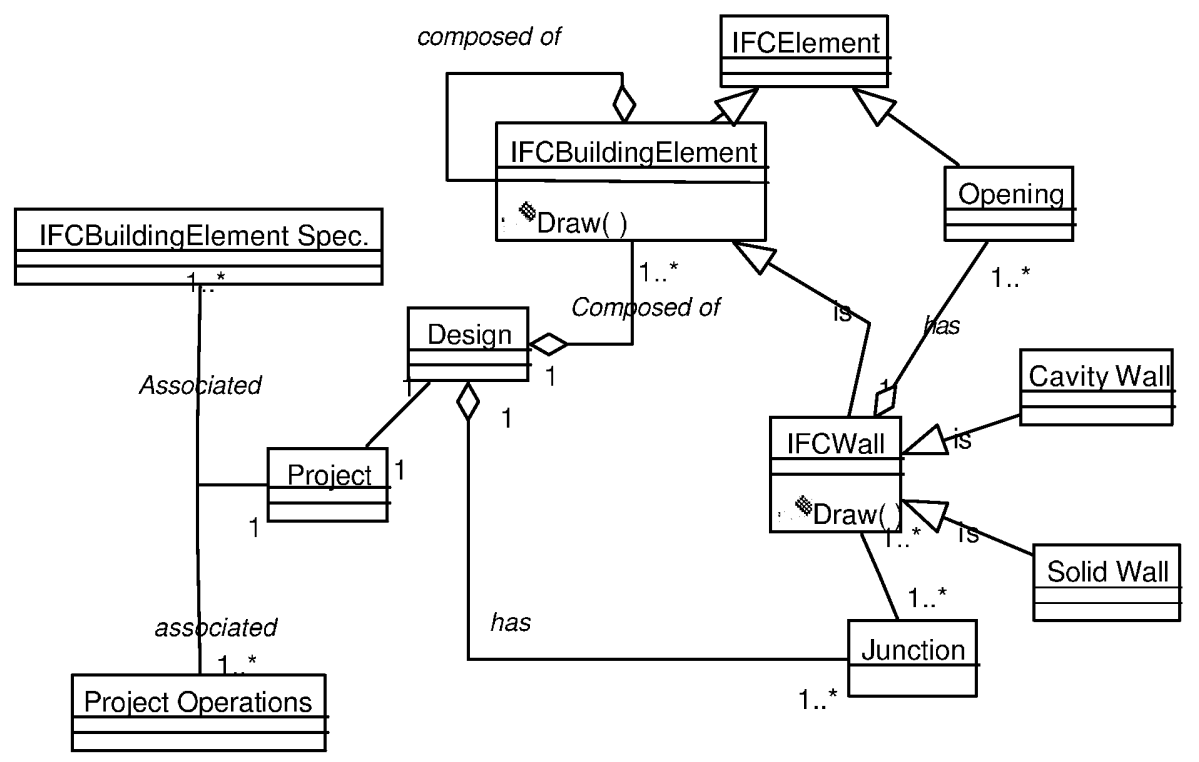

Fig. 2. A subset of the OSCONVR architectural design model.

\subsection{The OSCONVR Abstract Design Classes}

The OSCONVR model is designed to be generic and abstract enough to allow separation between the design model and the specific implementations of commercial CAD tools. For this it uses the Abstract Factory Design Pattern which provides an interface for creating families of related objects without specifying their concrete classes (Gamma E et al., 1994). As shown in Figure 3, the OSCONVR system defines:

- an Abstract ShapeFactory Class that declares the interface for creating each basic kind of design element,

- an Abstract Design Class (IfcBuildingElement) that represents an abstraction for each kind of architectural design element and provides abstractions that the OSCONVR design model classes can use to draw and render themselves in any graphical display environment

- a set of Concrete Subclasses of the ShapeFactory Class which implement the design elements for a specific CAD or graphical display environment. These subclasses can be used by the model classes to 
'draw' themselves without knowing which application is actually doing the drawing. In OSCONVR application the VRML_Drawer are the concrete classes designed specifically for the VRML applications respectively, and

- a set of client classes that encapsulate the commands the users will want to issue. Instances of these classes can then be used to implement command functionality in an environment independent manner.

The ShapeFactory's interface has an operation that returns a new design object for each Abstract Design Class of the design component. The client class (from the user application) uses the IfcBuildingElement Abstract Design Class, which calls operations to obtain instances of a design element and draw themselves without being aware of the Concrete Classes they are using. In other words, the client class has to commit to an interface defined by the Abstract Design Class, not to a particular Concrete Class.

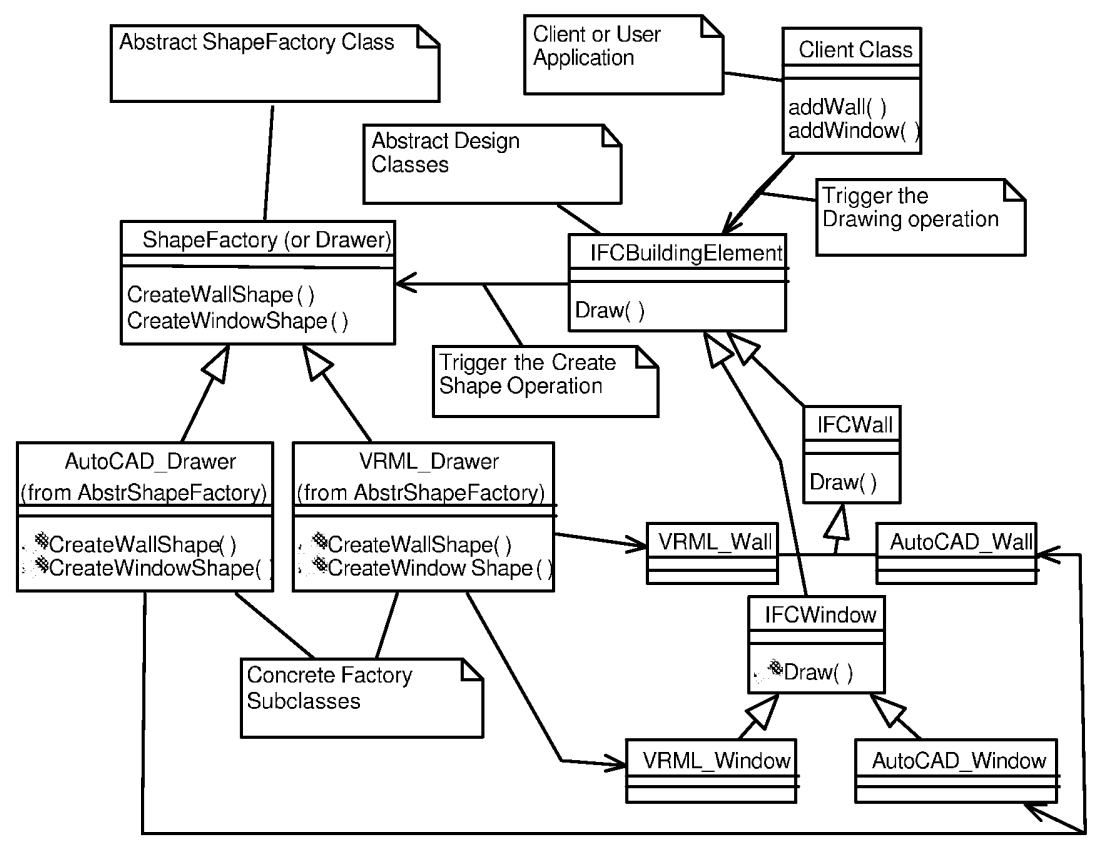

Fig. 3. The OSCONVR Abstract ShapeFactory and Abstract Design Mode

In practice, all classes in the OSCONVR design model inherit from the base class IFCBuildingElement that defines a common interface. This interface includes a $\operatorname{draw}()$ operation which is redefined in subclasses to allow instances of the design classes to draw themselves in a given display environment. The $\operatorname{draw}()$ operation passes a pointer to the ShapeFactory (or Drawer) object which encapsulates a set of simple 3D drawing operations as virtual member functions. These functions are implemented in the ShapeFactory concrete subclasses i.e. VRML_Drawer, to display 
the drawing in specific environments. As a result of this modelling approach the design of building components stay independent of the prevailing VR display environment and the VRML applications discussed below become graphical front ends for the instances of the OSCONVR architectural design model.

\section{The OSCONVR Prototype}

The Virtual Reality Modelling Language (VRML) is a developing standard for describing interactive three-dimensional scenes developed across the internet. A VRML browser is needed to load VRML files, which allow users to navigate through VRML worlds. The VRML file is a textual description of the VRML World. It contains nodes that describe shapes and their properties. VRML's four primitive shapes include cube, sphere, cone and cylinder. Figure 4 illustrates the overall architecture of the link between a VRML viewer and an integrated database

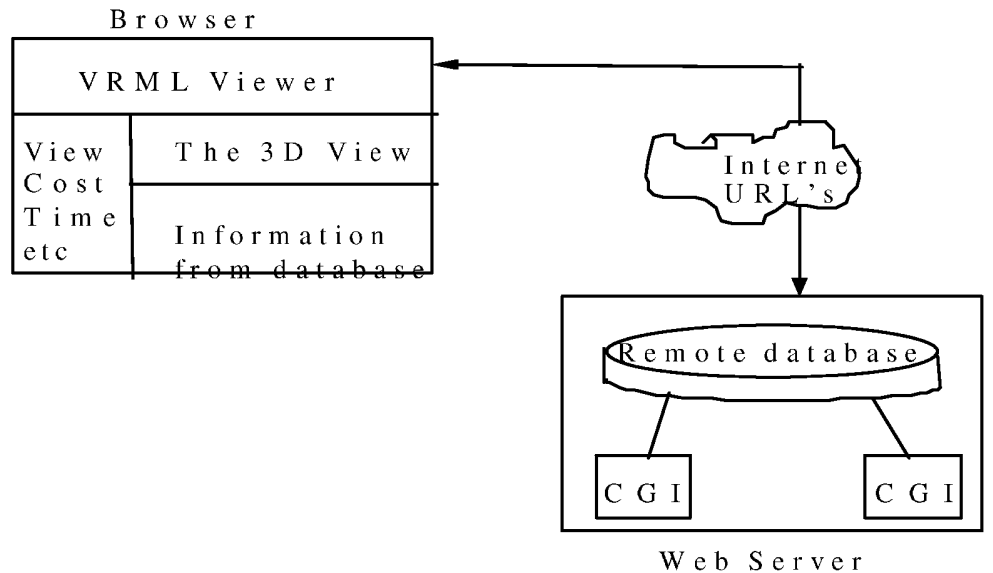

Fig. 4. The OSCONVR Architecture

The above figure shows diagrammatically how information is accessed from the database. The VRML standard allows links between different worlds to be established on the web. This puts the entire Internet at the fingertips of its users. Links are anchored to specific objects such as walls, beams, etc. By clicking on an anchored object you request information from other worlds using a URL(Universal Resource Locator) that specifies the address of a file on the web. World loaded from the Internet are delivered by the web server running on the remote host at the remote Internet site. In our case, the URL specifies a CGI script, which is a c++ program to run on the remote host under the control of the web server. The CGI program returns information about objects being queried in the VRML browser.

As previously mentioned, Virtual Reality has mostly been looked at as a visualisation tool. This paper puts the argument that VR should be used as the user interface. For instance, the user should interact with a $3 \mathrm{D}$ column in VR rather than a column in 
traditional database environment. This will allow the construction practitioners better access to information, which will motivate them to use integrated databases. In Figure 5 shown below, a screen shot of a house shown in VRML. The information is read from the OSCON object oriented database and displayed in this VR environment. The user can navigate inside the building clicking on design objects and retrieving information about their properties which include geometrical, cost and time data

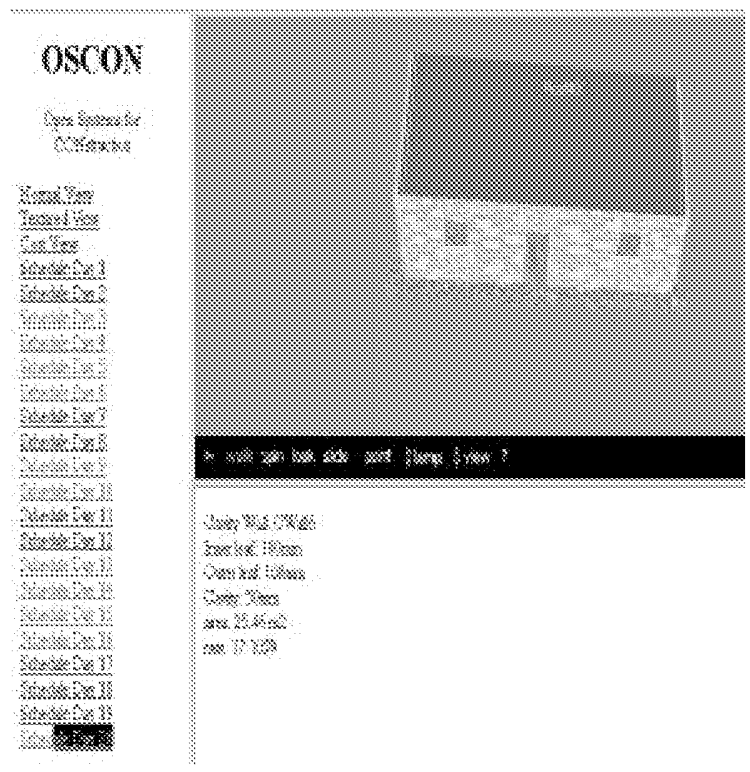

Fig. 5. An OSCONVR house model

The VRML environment to retrieve information is split into three windows using frames. The top window displays the VR representation of the building. This where the user interacts with the VRML image. The bottom windows display the information about objects queried in the VRML environment. The left-hand side windows include the views, which are supported by the system. These are described as follows:

- The normal view displays the basic representation of the building without any textures. It is mainly used for visualisation purposes.

- The textured view shows the textures associated with building elements such as walls, roofs, doors, etc..

- The cost view displays a coloured representation of the building according a colour criteria. For instance, an not estimated item or an expensive unit will appear in yellow. This would help to chase mistakes in the estimating model through the VRML environment.

- The planning view shows the various phases of the project plan in the $3 \mathrm{D}$ environment. For instance, in schedule day 1 only the slab is shown whereas in 
schedule day 5 the slab and cavity walls are shown according to information stored in the project plan.

It has to be said that there is not limit on the views, which the system can support. For instance, facilities management, structural design, or any other view can be added easily.

\section{How the System Works}

The user is provided with a suite of integrated applications, which support the design and time and cost planning of buildings. The AutoCAD design interface is used to generate the design layout of the building. The user interacts with AutoCAD as the graphical display environment. In reality, the design information is instantiated in the integrated object oriented database and displayed in AutoCAD. The design information is then used in generating quantities which are used by the time and cost planning prototype software. The VRML application is used to show the building in $3 \mathrm{D}$ and to retrieve information about specific objects in terms of cost, time, etc. The textures of design objects could be changed in the object-oriented database and the implications on cost and time are displayed within the VRML environment. This is one example of how design can be changed in the database by modifying its specifications and VR is used as the medium to show such implications. For a better description of the numerous functions supported by the OSCON database, the reader is referred to Aouad et al [3]. This direct interaction with a VR environment has many advantages over the use of a CAD package. In traditional CAD systems, the design is relatively static, which makes changes costly and time consuming as new images are re-generated from sequences of fixed frames [11]. In a VR environment, changes are handled efficiently by the technology. The ultimate benefit is the ability to create walkthroughs, which can facilitate collaboration between clients, designers, contractors and suppliers. This is the main objective of the OSCON database and it is strongly believed that VR is the medium for communication and convergence.

\section{Conclusions}

This paper discussed the development of a VRML prototype, which will be used as an interface for a project integrated database (OSCON). This prototype is a web-based application, which can be run from any web site. This will allow for construction information to be readily communicated between head offices and construction sites and any other locations. This will ensure that information is communicated in a much better format with a lot more visualisation capabilities. This paper has demonstrated that a VRML can be used as an interface to a complex object oriented database. This interface has more navigation capabilities. The user will ultimately find it simpler to navigate in a VR environment rather than browsing through thousands of records in a crude database environment. 
1. References Alshawi, $M$ \& Faraj, I. Integrating CAD and VR in construction. Proceedings of the Information Technology Awareness Workshop. January 1995, University of Salford.

2. Ames, A et al. The VRML source book, John Wiley and sons, 1996.

3. Aouad G., Marir F., Child T., Brandon P. and Kawooya A. (1997). A Construction integrated databases- Linking Design, Planning and Estimating. International Conference on Rehabilitation and Development of civil engineering infrastructure systems, June 9-11, 1997, American University of Beirut, Lebanon.

4. Griffin, M. Applications of VR in architecture and design. Proceedings of the Information Technology Awareness Workshop. January 1995, University of Salford.

5. http://www.construct.rdg.ac.uk/ITProjects/proje123.htm(Ashworth: linking Kappa, AutoCAd and WTK)

6. http://www.strath.ac.uk/Departments/CivEng/vesrg.html (Retik visual scheduling)

7. http://www.construct.rdg.ac.uk/ITsearch/Projects/project12.html(James Powell: visualisation)

8. http://wquoll.maneng.nott.ac.uk/Research/virart/industry/maintran.html(Nottingham, maintenance training: VIRART)

9. http://wquoll.maneng.nott.ac.uk/Research/virart/industry/rp.html(Nottingham,Rapid prototyping: VIRART)

10. Hubbold, R and Stone, R. Virtual reality as a design tool in Rolls Royce. Proceedings of the Information Technology Awareness Workshop. January 1995, University of Salford.

11. Larijani, L.C. The virtual reality primer. McGraw-Hill, USA, 1994.

12. Lorch, R. Animation in communication. Proceedings of the Information Technology Awareness Workshop. January 1995, University of Salford.

13. Penn, A et al. Intelligent architecture: rapid prototyping for architecture and planning. Proceedings of the Information Technology Awareness Workshop. January 1995, University of Salford.

14. Retik, A \& Hay, R. Visual simulation using VR. Arcom 10th conference, Vol 12, 1994. pp 537-546.

15. Svensson K. and Aouad G. (1997), Developing standardised architectural building product models for spaces and space enclosures. Submitted to Automation in Construction.

16. Froese T. and Paulson B. (1994). OPIS: An object model-based project information system. Microcomputers in Civil Engineering, 9, pp 13-28.

17. IFC (Industry Foundation Classes) (1997), Release 1.0, Vol 1-4. 